\title{
Validation of an automated technique for determining the mechanical characteristics of coronary arteries during balloon angioplasty: laboratory assessment with necropsy segments
}

\author{
A Murray, V Allen, M K Bennett
}

\begin{abstract}
Objectives-To develop a technique for automatic inflation of a percutaneous transluminal coronary angioplasty (PTCA) balloon, with continuous measurement of the balloon pressure and volume; to validate the technique for determining the mechanical characteristics of coronary arteries.

Methods-During necropsy examination of the hearts of nine patients, 17 coronary artery samples were obtained for histological examination. A PTCA balloon was inserted into each artery, and the balloon pressure and volume were measured continuously during four repeat automatic inflations of the balloon.
\end{abstract}

Results-Of the 17 arteries, eight showed elastic, six plastic, and three fracture pressure-volume deformation characteristics. For the plastic deformations, the first inflation required a higher pressure than subsequent repeat inflations of 82 (61) $\mathrm{kPa}$ (mean (SD), range 25 to $175 \mathrm{kPa}$ ). For the three in the fracture group, the pressure drop because of the fracture occurred between 210 and $540 \mathrm{kPa}$. Two of these three showed a tear on visual inspection, and the other showed disruption of the intimal plaque on blinded histological examination. Of the six with plastic deformation characteristics alone, one showed a tear, and on histological examination two others showed splitting of the internal and external elastic lamina and one showed separation of intima and media. None in the elastic group showed any of these characteristics.

Conclusions-Plastic and fracture deformation characteristics could be differentiated from elastic characteristics. Visual or histological evidence of fracturing was present in all three arteries identified during angioplasty as having pressurevolume fracture characteristics.

(Heart 1998;79:608-612)

Keywords: angioplasty; PTCA; coronary artery; pressure-volume deformation

Histopathology,

Freeman Hospital

M K Bennett

Correspondence to:

Professor Murray.

Accepted for publication 12 November 1997
Percutaneous transluminal coronary angioplasty (PTCA) is used extensively to treat patients with coronary artery stenosis. PTCA was pioneered by Gruentzig ${ }^{1}$ in the 1970s.
Using this technique a small balloon tipped catheter is positioned inside the artery, at the arterial stenosis, and the balloon inflated to dilate the stenosis. The technique is much less invasive than surgery, patients require less convalescence, and the procedure is considerably cheaper than coronary artery surgery.

Unfortunately, even when the direct effect of PTCA on the artery appears to be good, the long term outcome is not always successful. Between $30 \%$ and $40 \%$ of arteries restenose within about six months. ${ }^{2}{ }^{3}$ At present, although factors influencing outcome are poorly understood and restenosis is unpredictable, dissection plays a major role. Huber et al reviewed 691 cases of PTCA dissection and concluded that complex dissection was associated with low success rates. ${ }^{4}$ Waller et al found that in 130 necropsy studies, PTCA cracks and tears with flaps were implicated in $62 \%$ of early abrupt closure of the PTCA site. ${ }^{5}$ Also, Beatt et al discovered evidence that restenosis was linked to the amount of mechanical stretching of the stenosed vessel wall during PTCA. ${ }^{6}$ The relation between the final balloon pressure and lumen diameter, estimated from radiographic images, has been studied by a number of research workers. ${ }^{6-8}$ However $x$ ray angiography does not have the resolution to observe the small continuous changes during the inflation. Jain et al made qualitative observations about stretching, compaction, and cracking from balloon pressure and the displacement of the inflation plunger during manually controlled inflation, ${ }^{9}$ and this was subsequently followed up by Demer, ${ }^{10}$ who was able to estimate maximum volume changes in rabbit aortas using a special custom made catheter.

Intravascular ultrasound has made great advances and can show the extent of plaque, and plaque disruption as a result of the PTCA procedure. ${ }^{112}$ However, intravascular ultrasound techniques cannot yet be used to investigate the arterial wall simultaneously with PTCA balloon inflation.

Angioplasty can open out the arterial lumen in a number of different ways, from plastic deformation of the lesion to fracturing of hard plaque. Disruption of the arterial wall may lead to thrombus formation and reocclusion, ${ }^{13}$ and more extensive dissection may promote restenosis. ${ }^{14}$ The ability to monitor the deformation characteristics of the artery during angioplasty would promote research leading to improvements in the PTCA technique. 
Table 1 Mechanical and histological data

\begin{tabular}{|c|c|c|c|}
\hline Sample & $\begin{array}{l}\text { Pressure-volume } \\
\text { analysis }\end{array}$ & Visual findings & Histopathology \\
\hline \multicolumn{4}{|c|}{ Pressure-volume elastic characteristic } \\
\hline E1 & Elastic & & Plaque to internal elastic lamina \\
\hline E2 & Elastic & & Plaque to internal elastic lamina \\
\hline E3 & Elastic & & Plaque ruptured internal elastic lamina \\
\hline E4 & Elastic & & Plaque ruptured internal elastic lamina \\
\hline E5 & Elastic & & Plaque ruptured internal elastic lamina \\
\hline E6 & Elastic & & Plaque ruptured internal elastic lamina \\
\hline E7 & Elastic & & Plaque to internal elastic lamina, $>50 \%$ circumference \\
\hline E8 & Elastic & & Plaque to internal elastic lamina \\
\hline \multicolumn{4}{|c|}{ Pressure-volume plastic characteristic } \\
\hline P1 & Plastic $25 \mathrm{kPa}^{\star}$ & & Plaque superficial \\
\hline P2 & Plastic $30 \mathrm{kPa} \star$ & & Plaque splitting of internal and external elastic lamina, $50 \%$ circumference \\
\hline P3 & Plastic $45 \mathrm{kPa}^{\star}$ & & Plaque splitting of internal and external elastic lamina, $25 \%$ circumference \\
\hline $\mathrm{P} 4$ & Plastic $80 \mathrm{kPa}^{\star}$ & & Plaque $50 \%$ circumference, intima and media separated \\
\hline P5 & Plastic $175 \mathrm{kPa} \star$ & & Plaque to adventitia \\
\hline P6 & Plastic $135 \mathrm{kPa}^{\star}$ & $7 \mathrm{~mm}$ linear tear & Plaque to external elastic lamina with multifocal involvement \\
\hline \multicolumn{4}{|c|}{ Pressure-volume fracture characteristic } \\
\hline $\mathrm{F} 1$ & Fracture $210 \mathrm{kPa} \dagger$ & $10 \mathrm{~mm}$ linear tear & Plaque $>75 \%$ circumference, calcified, fractured \\
\hline F2 & Fracture $230 \mathrm{kPa} \dagger$ & & Plaque ulcerated, disruption of intimal plaque \\
\hline F3 & Fracture $540 \mathrm{kPa} \dagger$ & Tear & Plaque to external elastic lamina, calcified \\
\hline
\end{tabular}

${ }^{\star}$ Maximum pressure difference between first and subsequent inflations; tpressure at which fracture occurred.

However, no technique has yet been made available for studying the continuous pressurevolume relations in detail using a standard PTCA catheter. This is perhaps not surprising as a very small volume change of the order of only $0.3 \mathrm{ml}$ is all that is required, and inflation and deflation must be finely controlled. In the medical physics department in Freeman Hospital we have developed a PTCA inflation system with quantitative measurement of the pressure-volume relations of arterial segments during PTCA, and have shown its ability to make highly repeatable measurements in laboratory assessments. ${ }^{15}{ }^{16}$ However, before such a system can be used clinically, its ability to detect measurable changes in arteries must be demonstrated.

The aim of the research reported here was to assess the practicality of measuring changes in non-elastic arterial properties with repeat inflations of an angioplasty balloon, and to confirm that these measurements relate to specific arterial wall damage observed macroscopically or histologically.

\section{Methods}

INFLATOR AND BALLOON CATHETER

Precisely controlled automatic inflation and deflation was achieved using a specially developed device. ${ }^{15}$ The PTCA balloon was inflated and deflated at a steady rate under electronic stepper motor control. A volume change rate of $0.05 \mathrm{ml} / \mathrm{s}$ was used, with a Schneider $10 \mathrm{ml}$ inflator/deflator (Schneider Co, Buelach, Switzerland). The volume change was given as an analogue output from the electronic control circuitry. Pressure was measured with a pressure transducer calibrated to $1 \mathrm{MPa}(10 \mathrm{bar})$ and a pressure amplifier. Any PTCA balloon catheter can be attached to the system. In the research described, an Advanced Cardiovascular Systems ACX coronary dilatation catheter was used (ACS Guidant, Santa Clara, California, USA). This has a stated balloon diameter of $3.5 \mathrm{~mm}$ at $6 \mathrm{bar}$.
MEASUREMENT SYSTEM

The PTCA inflator was filled with water and attached to a $2 \mathrm{~m}$ length of connecting tube (monitoring/infusion line, low density polyethylene, inner diameter $1 \mathrm{~mm}$ ). The long $2 \mathrm{~m}$ length of tube was used as it represented the longest length required for subsequent clinical studies. Also, our previous work ${ }^{15}{ }^{16}$ showed that the effect of this length was negligible in comparison with other properties, and that the use of water produced results indistinguishable from those with contrast because of the very low flow rates used. The other end of the tube was connected to a three way tap, which was in turn connected to a fluid reservoir (for filling the system) and a second three way tap. This tap was connected directly to the pressure transducer and PTCA catheter. Before the transducer and catheter were connected they were filled carefully to remove all visible traces of air. To aid this, the PTCA catheter was put under negative pressure several times for several seconds, as it would be during a normal clinical procedure. All joints were carefully tightened to ensure that they would not leak, and after confirming by inspection that there were no visible air bubbles in the system, the tap to the reservoir was turned off.

\section{CORONARY ARTERIES}

At necropsy, coronary arteries were obtained and these included epicardial fat by dissection from the myocardium. Twenty one segments were obtained from nine hearts. Four of these were subsequently found to be completely occluded and the PTCA balloon could not be inserted. Of the remaining 17,12 were at least $25 \mathrm{~mm}$ long, with the other five were between 20 and $10 \mathrm{~mm}$.

\section{MEASUREMENT PROTOCOL}

The arterial sample was placed in a shallow dish filled with normal saline. The deflated balloon was inserted carefully into the artery. For short segment lengths, it was positioned centrally. The balloon was then inflated automatically to $800 \mathrm{kPa}$ ( 8 bar) and after a 

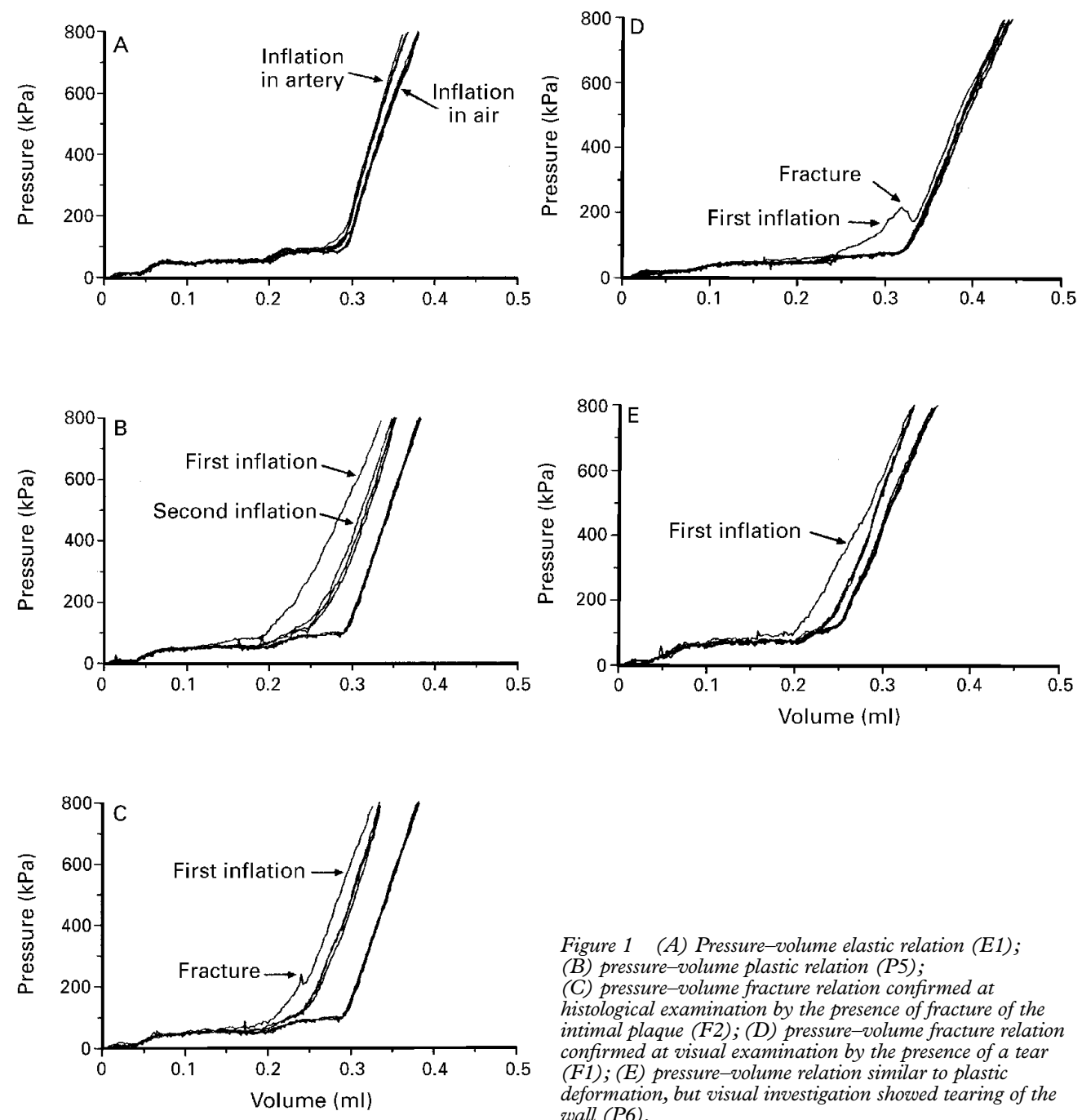

Figure 1 (A) Pressure-volume elastic relation (E1); (B) pressure-volume plastic relation (P5); (C) pressure-volume fracture relation confirmed at histological examination by the presence of fracture of the intimal plaque (F2); (D) pressure-volume fracture relation confirmed at visual examination by the presence of a tear (F1); (E) pressure-volume relation similar to plastic deformation, but visual investigation showed tearing of the wall (P6).

delay of 10 seconds deflated. This was repeated four times. The position of each angioplastied section was measured and noted for the subsequent macroscopic and histological examination. The balloon was then removed from the artery and inflated/deflated four times in air.

PATHOLOGY EXAMINATION

Immediately after the PTCA, the artery was opened longitudinally and carefully examined for areas of stenosis and tears. Histological sections were then prepared by fixing the tissue in $4 \%$ buffered neutral formalin for at least two days, followed by decalcification. Areas of interest were then serially sliced and paraffin blocks made. Each was then sectioned at $4 \mu \mathrm{m}$ and routinely stained with haematoxylin and eosin, and the arterial elastic tissue visualised by Verhoeff's method. Care was taken during the subsequent microscopic examination to avoid listing artefactual features which might have been produced in the preparation of the sections. Each of the arteries was assessed, blind to angioplasty results, for the type of damage, the depth of fibrous plaque, and the presence of calcified plaque.

\section{Results}

A summary of the pressure-volume data, visual inspection, and histological data in the 17 coronary arteries studied is given in table 1 . In eight arteries there were no quantifiable differences in the pressure-volume characteristics between any of the four inflations, showing that the arteries retained their characteristics from one inflation to the next and could therefore be characterised as elastic (E). An example of one (E1; numbering refers to the order in table 1$)$ is given in fig 1A, showing the similar characteristics for each of the four repeat inflations of the balloon in the artery. This panel, and the subsequent panels, also shows the four repeat inflation cycles in air, superimposed on each other. The difference between inflation in air and inflation in a coronary artery results from the properties of the coronary artery.

In the other nine arteries, the pressurevolume characteristics were very different in the first inflation than in the subsequent three inflations. In all cases, the indication was that the deformation occurred during the first inflation. In six, the pressure continued to rise throughout the inflation, while in the other three the pressure suddenly fell during inflation 

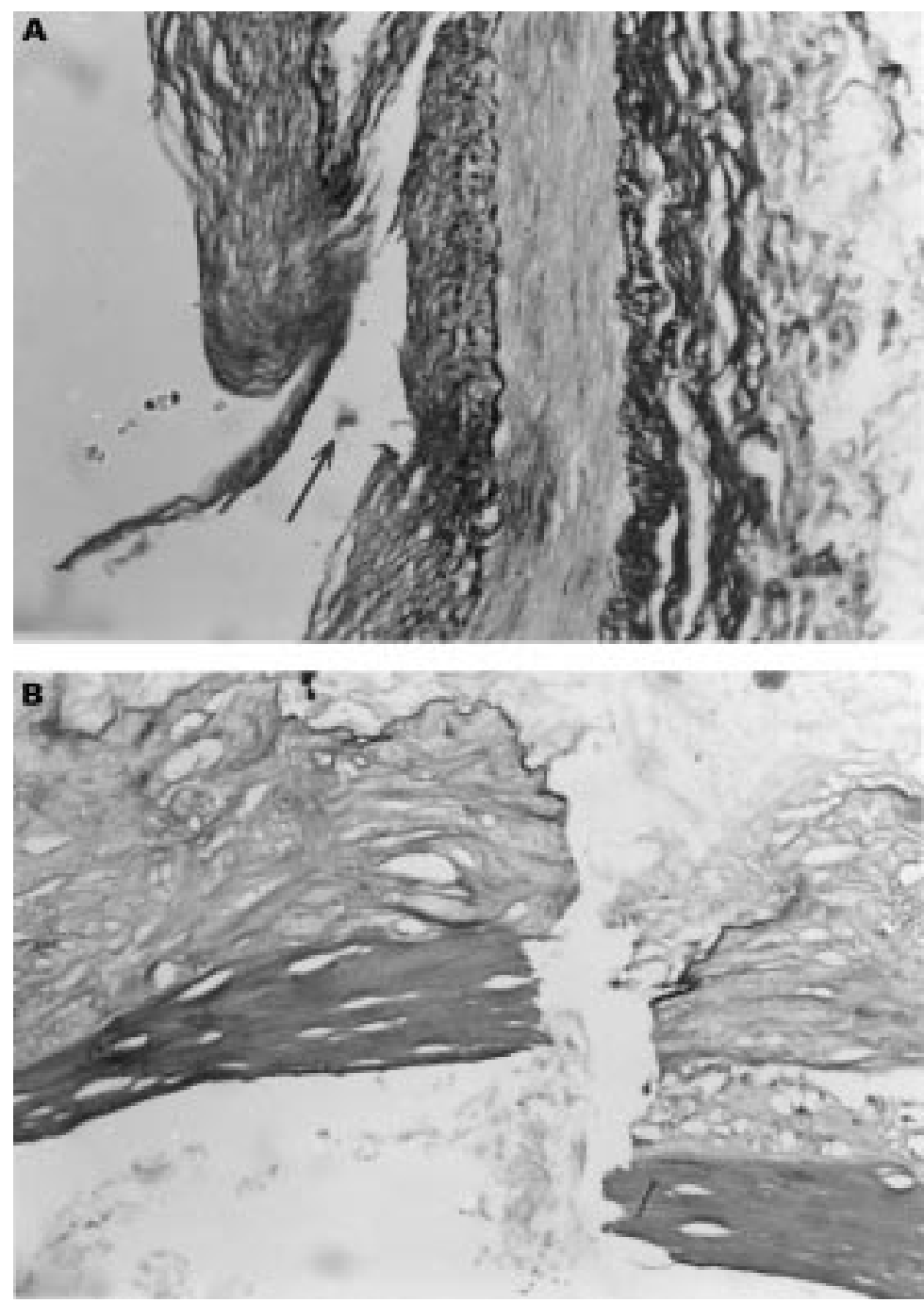

Figure 2 (A) Histological section of artery with pressure-volume fracture, showing disruption of intimal plaque (F2). (Original magnification $\times 32$. Stained for elastic tissue by Verhoeff's method.) The arrow points to the intimal tear. (B) Histological section of artery with pressure-volume fracture, showing fracture of the heavily calcified intima above an atheromatous plaque (F1). (Original magnification $\times 32$. Stained by haematoxylin and eosin.)

before continuing to rise; the six illustrate only plastic (P) deformation, and the other three illustrate mechanical failure of the arterial wall structure and are classified as fracture (F) deformation. An example of substantial plastic deformation (P5) is shown in fig $1 \mathrm{~B}$, and in this case the second inflation continued to produce a small amount of plastic deformation. Examples of fracture deformation are given in figs $1 \mathrm{C}$ and $\mathrm{D}$. That in fig $1 \mathrm{C}$ shows a small but quickly propagating fracture $(\mathrm{F} 2)$, while that in fig $1 \mathrm{D}$ shows a much larger but more slowly propagating fracture $(\mathrm{F} 1)$.

Examination of the arteries was undertaken blind to the knowledge of any pressure-volume information. Visual inspection of the arteries after the procedure indicated that three had suffered obvious fracture, two in the pressurevolume fracture group and one in the pressurevolume plastic group. This latter (P6, fig 1E) showed no major pressure-volume difference from the others in the plastic group, indicating that the tearing of the artery occurred gradu- ally with the increasing balloon inflation, and there was no sudden failure.

Histological examination of the arteries confirmed that plaque was present in all of them, but was more extensive in the plastic and fracture groups (table 1). A histological section of the artery in our fracture group which had no visible evidence of failure showed disruption of the intimal plaque (F2, fig 2A). A similar histological section in the artery with the large slowly propagating fracture clearly showed fracture of the heavily calcified plaque (F1, fig 2B). Of the five arteries in our plastic group without direct visual evidence for the permanent deformation, one had separation of the intima and media, and two others had evidence of splitting of the internal and external elastic lamina. None of the eight in the elastic group showed visual or histological evidence of any permanent deformation.

\section{Discussion}

Much of the early research in examining pressure-volume relations used indirect measurement of volume, such as from angiographic images. ${ }^{7}$ The technique assessed here is able to record volume directly and continuously.

We have previously shown that in simulations using small bore elastic tubes the pressure-volume curves are highly repeatable, with a standard deviation error from the mean of only $9.0 \mathrm{kPa} .{ }^{15}$ Eight of the arterial samples showed tight grouping of the four repeat measurement cycles. All the others showed a clear deviation of the first pressure-volume inflation curve from the curves of the subsequent inflations. For the six in our plastic deformation group, the differences ranged from 25 to $175 \mathrm{kPa}$ (mean (SD), 82 (61) $\mathrm{kPa}$ ), well outside our repeatability error. In five of these six, the plastic deformation had been achieved in the first inflation. There was some evidence that in the artery with the greatest deformation (P5, fig 1B) there was some additional deformation on the second inflation, but this was much smaller than for the first.

The pressure-volume curves clearly identified fracture deformation. There was, however, one case in which fracture could not be differentiated from plastic deformation, and this could have been caused by the fracture propagating slowly as the balloon was inflated. In the three with pressure-volume fracture, the balloon pressure at which this occurred could be identified clearly (table 1). The balloon pressure results from stress in the balloon wall as well as in the arterial wall, and during clinical angioplasty it is not possible to differentiate between the two. However, with our technique it is possible to assess the characteristics of the balloon before inserting it into the coronary artery, and it is hence possible to identify separately the stress being produced in the arterial wall. It should therefore be possible to prevent excessive stresses which might lead to permanent undesirable fracture or dissection.

Continuous pressure-volume measurements were obtained by Jain et al using manual inflation to enable qualitative observations to be 
made, ${ }^{9}$ and this was followed up by Demer ${ }^{10}$ in the same centre, using a specially designed balloon catheter in rabbit aortas. Our report is the first to provide such detailed quantitative pressure-volume curves using automatic inflation with a standard angioplasty inflator and balloon catheter in human arteries.

Preparation of samples for histological examination can introduce distortion in the samples, and hence we have reported only those observations which could not have arisen artificially. Because of the extensive disruption of the arteries by plaque we were unable to use histology to differentiate those arteries more likely to result in the different deformation characteristics.

We accept that this technique has limitations and that the samples used in this study may not give the same results as those from intact arteries, since the cut ends could make fracture more likely. The effect of the balloon and the inflation system is superimposed on that of the artery, and hence the technique requires a calibration inflation of the balloon in air. It was not our intention in this paper to uncover new data on arteries, but to demonstrate a practical measurement technique which can be used during clinical PTCA. We have shown that it is possible to identify different arterial deformation characteristics from coronary artery angioplasty pressure-volume curves. Research is now required to assess the ability of our technique to identify those angioplasty procedures that are likely to result in arteries remaining patent for longer. The technique has potential for influencing the way clinical angioplasty is carried out.
This work was supported by the British Heart Foundation.

1 Gruentzig AR. Transluminal dilatation of coronary artery stenosis. Lancet 1978;i:263.

2 Meier B. Restenosis after coronary angioplasty: review of the literature. Eur Heart f 1988;9(suppl C): 1-6.

3 Levine GN, Chodos AP, Loscalzo J. Restenosis following coronary angioplasty: clinical presentations and therapeutic options. Clin Cardiol 1995;18:693-703.

4 Huber MS, Mooney JF, Madison J, et al. Use of a morphologic classification to predict clinical outcome after dissection from coronary angioplasty. Am $\mathcal{F}$ Cardiol 1991;68:46771

5 Waller BR, Fry ETA, Peters TF, et al. Abrupt ( $<1$ day), acute ( $<1$ week), and early ( $<1$ month) vessel closure at the angioplasty site. Morphologic observations and causes of closure in 130 necropsy patients undergoing coronary angioplasty. Clin Cardiol 1996;19:857-68.

6 Beatt KJ, Serruys PW, Luijten HE, et al. Restenosis after coronary angioplasty: the paradox of increased lumen coronary angioplasty: the paradox of increased lumen

7 Hjemdahl-Monsen CE, Ambrose JA, Borrico S, et al. Angiographic patterns of balloon inflation during percutaneous transluminal coronary angioplasty: role of pressurediameter curves in studying distensibility and elasticity of the stenotic lesion and the mechanism of dilation. $\mathcal{F} \mathrm{Am}$ Coll Cardiol 1990;16:569-75.

8 Rensing BJ, Hermans WR, Strauss BH, et al. Regional differences in elastic recoil after percutaneous transluminal coronary angioplasty: a quantitative angiographic study. $\mathcal{F}$ Am Coll Cardiol 1991;17:34-8b.

9 Jain A, Demer LL, Raizner AE, et al. In vivo assessment of vascular dilatation during PTCA. Am f Cardiol 1987;60: 988-92.

10 Demer L. Effect of calcification on in vivo mechanical response of rabbit arteries to balloon dilatation. Circulation response of rabbit

11 Marsico F, Kubica J, De Servi S, et al. Influence of plaque morphology on the mechanism of luminal enlargement after directional coronary atherectomy and balloon angioplasty. Br Heart f 1995; 74:134-9.

12 Jeremias A, Ge J, Erbel R. New insight into plaque healing after plaque rupture with subsequent thrombus formation detected by intravascular ultrasound. Heart 1997;77:293.

13 Essed CE, van den Brand $M$, Becker AE. Transluminal coronary angioplasty and early restenosis; fibrocellular
occlusion after wall laceration. Br Heart $\mathcal{f} 1983 ; 49: 393-6$.

14 Pathology of coronary angioplasty. Lancet 1989;ii:423-4. Murray A, Allen V. Angioplasty pressure-vo
measurement. Med Biol Eng Comput 1995;33:747-8.

16 Murray A, Allen V. Measurement of angioplasty lumen volume and wall compliance: a laboratory study. Physiol Meas 1997;18:39-47. 